\title{
Bone Marrow Hyperplasia in Elderly Acute Lymphoblastic Leukemia Changed From Low Into Hyperactive: a Case Report
}

\section{Bin Zhang ( $\sim$ zhangb2021cn@126.com )}

the first affiliated hospital of hebei north university

\section{Yan-Xia Cai}

the first affiliated hospital of hebei north university

\section{Xia Wang}

the first affiliated hospital of hebei north university

Xun-Tao Liu

the first affiliated hospital of hebei north university

Sheng-Chun Fang

the first affiliated hospital of hebei north university

Su-Zhen Zhang

the first affiliated hospital of hebei north university

Fei Zhao

the first affiliated hospital of hebei north university

\section{Di-Yuan Guo}

the first affiliated hospital of hebei north university

\section{Li-Na Geng}

the first affiliated hospital of hebei north university

\section{Case Report}

Keywords: bone marrow hypoplastic, hyperactive, B lymphocytic leukemia, morphology, biopsy

Posted Date: May 11th, 2021

DOI: https://doi.org/10.21203/rs.3.rs-506318/v1

License: (c) (1) This work is licensed under a Creative Commons Attribution 4.0 International License. Read Full License 


\section{Abstract}

Background: To retrospectively reported a case of bone marrow hyperplasia in elderly acute lymphoblastic leukemia changed from low into hyperactive, in order to improve the understanding of this disease.

Case presentation: We continuously followed up a patient of bone marrow hypoplastic patients by evaluating his clinical results, bone marrow morphology and flow cytometry, and analyzing the results at different locations in two consecutive bone marrow punctures. The patient's hypoplastic bone marrow suddenly became extremely hyperplasia. Before this change, there was early-stage alterations in morphology, flow cytometry and biopsy of the bone marrow, but there was no significant change in peripheral blood cells.

Conclusions: For patients whose hypoplastic bone marrow suddenly became extremely hyperplasia, morphology was not very useful, thus definite diagnosis must be dependent on immunological types and bone marrow biopsy results.

\section{Background}

Bone marrow hypoplasia is mainly seen in acute arrest of hemopoiesis and in aplastic anemia [1]. Aplastic anemia is a hematopoietic function failure syndrome caused by many potential factors, and is characterized by lack of hematoblasts in the bone marrow and the reduced peripheral blood cells, and its clinical manifestations include anemia, hemorrhage and infection [2]. Although its pathogenesis is not fully understood, but aplastic anemia is confirmed to be related to chemicals, radiation, virus infection and genetic factors [2]. It has two peak periods of incidence, i.e., 15-25 years of age and $>60$ years of age [2]. According to the extent of bone marrow failure and the progression of clinical symptoms, it can be categorized as sever and non-severe, or acute and chronic [3].

Lymphocytic leukemia is a clonal malignant disease originate from lymphocytes, with an incidence rate of 2.7/100, 000 [4]. It has a rapid onset, and clinical symptoms are related to the hyperplasia and infiltration of lymphocytes, especially in elderly patients, whose immune functions are worse and thus prognosis is bad [5]. The manifestations of bone marrow include extreme hyperplasia, and is mainly comprised of primitive cells, which is different from aplastic anemia and can be used to distinguish it from aplastic anemia [6]. The percentage of primitive cells should be $>20 \%$ in lymphocytic leukemia [6]. However, in clinic, if the main cells are lymphocytes in peripheral blood smear or bone marrow biopsy, it will often be diagnosed as bone marrow hypoplasia or aplastic anemia, especially in morphology tests, due to the darker staining of the cells, the primitive cells are hardly to be distinguished by microscopy [7]. Therefore, we need to find a better way to accurately diagnose these diseases.

In our recent works, we identified a case of B-lymphocytic leukemia, which was firstly though to be aplastic anemia based on morphology results at two different locations but was evolved to extremely 
hyperplasia after 50 days. We hope that this report can provide evidence and suggestions for the accurate diagnosis of these diseases.

\section{Case Presentation}

\section{Clinical manifestations of the patient}

The current study has been approved by the Ethics Committee of our hospital, and the patientd signed written informed consent.

The patient was female and aged 71. Her main complaints were fatigue, short breath and inappetence. Physical examinations showed anemic appearance, apparent sternal tenderness, but without stained yellow or hemorrhagic spot in her skin or mucosa. There was an enlarged, firm lymph node in the right neck sized about $1 * 1 \mathrm{~cm}$, with the absence of tenderness and a low mobility. No other lymph nodes were detected.

2. Lab equipment and reagents

Blood routine tests were done in XN3000 blood cell counter (SYSMEX, Japan). Flow cytometry was done in BD FACSCalibur and analyzed by CELLquest software. Antibodies, including anti-CD3, anti-CD2, antiCD5, anti-CD7, anti-CD19, anti-CD20, anti-CD22, anti-CD9, anti-CD23, anti-CD71, anti-CD14, anti-CD13, anti-CD33, anti-CD34, anti-CD38, anti-HLA-DR, anti-MPO, anti-CD79a, anti-CD64, anti-CD11b, anti-CD10, anti-CD25, anti-Kappa and anti-Lambda were all obtained from Becton Dickson (USE). BX53 optic microscope was bought from OLYMPUS (Japan). POX staining reagent and Giemsa staining reagent were bought from Beisuo biotechnology company (Zhuhai, China).

3. Morphology tests of bone marrow and peripheral blood cells

Freshly obtained smears of bone marrow and peripheral blood cells were stained in room temperature for 20 minutes and then observed under the optic microscope.

4. Determination of immunological types

Before treatment, 1.5-2.0 mL of bone marrow was anticoagulated by EDTA, and then immumofluorescence was used to detect the expression of CD45percp, CD5 APC, CD2 PE CD3 APC, CD19 PE, CD22PE, CD20 FITC, CD34 FITC, CD38 PE, CD10 FITC, CD25 PE, TdT FITC, CD7 FITC, CD56 APC, CD79a PE, HLA-DR, CD13 PE, CD4 FITC, CD8 PE, CD64 PE, CD14 FITC, MPO FITC, CD9 FITC, CD23 PE, CD15 FITC, CD117 PE, Kappa FITC and Lambda PE.

\section{Results}

1. Peripheral blood cells 
As shown in Fig. 1, from the first test on Jan.26, 2018 to the last test on Mar.7, 2018, peripheral white blood cells (WBC), red blood cells (RBC), hemoglobin (HGB) and platelet (PLT) increase to normal level, meanwhile lymphocytes percentage decreased to normal, and segmented neutrocyte percentage increased to normal, while there was no change in monocyte percentage(Table 1).

Table 1

Peripheral blood cells

\begin{tabular}{|c|c|c|c|c|c|c|c|}
\hline $\begin{array}{l}\text { Examined } \\
\text { date }\end{array}$ & $\begin{array}{l}\text { WBC } \\
\left(10^{9} / L\right)\end{array}$ & $\begin{array}{l}\text { RBC } \\
\left(10^{12} / \mathrm{L}\right)\end{array}$ & $\begin{array}{l}\text { HGB } \\
(\mathrm{g} / \mathrm{L})\end{array}$ & $\begin{array}{l}\text { PLT } \\
\left(10^{9} / L\right)\end{array}$ & $\begin{array}{l}\text { Lymphocyte } \\
\text { percentage } \\
\text { (\%) }\end{array}$ & $\begin{array}{l}\text { Segmented } \\
\text { neutrocyte } \\
\text { percentage (\%) }\end{array}$ & $\begin{array}{l}\text { Monocyte } \\
\text { percentage } \\
(\%)\end{array}$ \\
\hline 20180126 & 3.07 & 2.31 & 72 & 14 & 83.1 & 6.8 & 7.8 \\
\hline 20180127 & 1.97 & 1.98 & 58 & 13 & 90.4 & 5.6 & 1.5 \\
\hline 20180131 & 1.14 & 1.85 & 57 & 23 & 91.2 & 3.5 & 2.6 \\
\hline 20180202 & 1.07 & 2.07 & 63 & 15 & 71.0 & 23.5 & 3.7 \\
\hline 20180204 & 1.37 & 2.3 & 69 & 48 & 44.5 & 49.7 & 5.8 \\
\hline 20180206 & 2.17 & 2.23 & 69 & 41 & 44.2 & 52.5 & 2.8 \\
\hline 20180208 & 2.71 & 1.89 & 58 & 46 & 25.8 & 71.2 & 2.2 \\
\hline 20180210 & 4.16 & 2.65 & 80 & 73 & 21.2 & 76.6 & 2.2 \\
\hline 20180212 & 3.26 & 2.52 & 75 & 106 & 30.1 & 68.1 & 1.2 \\
\hline 20180214 & 2.48 & 2.46 & 74 & 155 & 37.5 & 60.1 & 1.6 \\
\hline 20180217 & 3.69 & 2.7 & 79 & 219 & 31.4 & 66.8 & 0.8 \\
\hline 20180220 & 4.57 & 2.81 & 81 & 261 & 17.1 & 81.2 & 0.2 \\
\hline 20180223 & 4.19 & 3.17 & 92 & 327 & 40.6 & 56.1 & 1.2 \\
\hline 20180226 & 4.29 & 3.25 & 94 & 268 & 40.8 & 55.9 & 1.2 \\
\hline 20180301 & 5.91 & 3.3 & 98 & 221 & 34.5 & 63.0 & 0.8 \\
\hline 20180304 & 6.75 & 3.65 & 107 & 180 & 31.7 & 65.5 & 1.0 \\
\hline 20180307 & 6.71 & 3.68 & 111 & 130 & 35.0 & 61.1 & 1.8 \\
\hline 20180310 & 5.19 & 3.55 & 106 & 98 & 37.4 & 58.8 & 1.7 \\
\hline 20180313 & 6.1 & 3.79 & 113 & 78 & 48.2 & 48 & 1.5 \\
\hline 20180315 & 5.98 & 3.75 & 113 & 74 & 50.2 & 46.8 & 1.2 \\
\hline
\end{tabular}




\section{Smear result of peripheral blood cells}

The smear of peripheral blood cells was done on Jan.27, 2018, which showed that segmented neutrocytes accounted for $8 \%$, and mature lymphocytes accounted for $86 \%$, atypical lymphocytes accounted for $6 \%$, and there were few platelets, and there were 8 nucleated red blood cells (NRBC).

\section{Smear result and genetic result of bone marrow}

Figure $1 \mathrm{~A}$ is the first bone marrow smear result done Jan.27, 2018. It shows bone marrow hypoplasia, and mature lymphocytes accounted for $96 \%$, segmented neutrocytes accounted for $1 \%$, NRBC accounted for $3 \%$. There was no megakaryocyte and there were few platelets.

Figure 1B is the second bone marrow smear result done Feb.5, 2018. It still shows bone marrow hypoplasia. Mature lymphocytes accounted for $39 \%$ (suspected juvenile cells in the entire smear sample were $0.5 \%$ ), neutrophilic myelocytes accounted for $1.5 \%$, neutrophilic metamyelocytes accounted for $1.5 \%$, band neutrophils accounted for $1.5 \%$, segmented neutrocytes accounted for $34 \%$, NRBC accounted for $21 \%$. There was one megakaryocyte and there were few platelets.

Figure $1 \mathrm{C}$ is the third bone marrow smear result done Mar.7, 2018. It shows extremely hyperplasia of the bone marrow. primitive lymphocytes accounted for $87 \%$, mature lymphocytes accounted for $4 \%$, neutrophilic metamyelocytes accounted for $0.5 \%$, band neutrophils accounted for $0.5 \%$, segmented neutrocytes accounted for $0.5 \%$, NRBC accounted for $7 \%$. There were 56 megakaryocyte and there were few platelets. Figure 1D is the POX staining which shows negative.

Genetic results show that it was BCR/ABL (P210) fusion gene positive.

\section{Bone marrow biopsy}

As shown in Fig. 2A, in the test done on Feb.5, 2018, abnormal cells accounted for $0.69 \%$, expression of CD45percp was weak, while SSC was slightly strong. Expression of CD34 (27.48\%), CD38 (40.68\%区, HLADR (16.42\%区, CD71 (31.50\%区, cCD79a (64.31\%匹was detected. CD2, CD3, CD5, CD19, CD10, CD20, CD22, CD9, CD4, CD8, Kappa, Lambda, CD56, CD117, CD7, CD13, CD33, CD64, CD14, MPO, CD23 was not expressed.

Figure 2B is the test done on Mar.10, 2018. It shows that abnormal cells accounted for $81.71 \%$, CD45percp was not expressed, and SSC was weakly expressed. Expression of CD34, CD38, CD19, CD10, CD9, CD22, HLA-DR, CD71, CCD79a, CD5 was detected. CD2, CD3, CD5, CD20, CD4, CD8, Kappa, Lambda, CD56, CD117, CD7, CD13, CD33, CD64, CD14, MP0, CD23 was not expressed. Thus, it was considered to be acute b-lymphocytic leukemia.

\section{Treatment and outcomes}

COP chemotherapy regimen was adopted, which included cyclophosphamide ( $0.8 \mathrm{~g}$ at day 1$)$, vincristine (2 mg at day 1 ), prednisone (60 mg from day 1 to day 7 ). During the chemotherapy, peripheral blood cells 
decreased, as WBC was 0.9×109/L, PLT was 0-2.0×109/L, and HGB was 59-67 g/L. Suspended red blood cells and platelets were infused, but no significant effects. At the last day of chemotherapy, infection occurred, which was not relieved even after a week of anti-infection of high generation antibiotics. Heart failure also occurred. The patient's family decided to stop treatment. Prognosis was poor, which was in accordance with the literature [3].

\section{Discussion}

From the first test on Jan.26, 2018 to the last test on Mar.7, 2018, peripheral white blood cells (WBC), red blood cells (RBC), hemoglobin (HGB) and platelet (PLT) increase to normal level, meanwhile lymphocytes percentage decreased to normal, and segmented neutrocyte percentage increased to normal, while there was no change in monocyte percentage. The results showed improved peripheral blood cell results. But as the later results showed, the patient got worse, thus it cannot be determined whether the disease was recovering or worsening by only referring to peripheral blood cell results. So we should be aware that not all leukemia patients' status can be reflected by peripheral blood cell results, if there are any suspensions, smear should be performed to further explore the causes of the disease [8-10]. Meanwhile, it should be noticed that the blood cell counter used in the clinical should be improved to be more sensitive to detect the juvenile cells.

The diagnosis of aplastic anemia should include decrease of all peripheral blood cells, bone marrow hypoplasia, and should also exclude other disease that can cause decreased peripheral blood cells (e.g., paroxysmal nocturnal hemoglobinuria, myelodysplastic syndrome, myelofibrosis, etc.), and the main cell type should he lymphocytes [11-13]. But there's no clear guidance on the number of juvenile cells, especially in bone marrow hypoplasia, and there's no requirement to include the number of nucleated cells, which could result in misdiagnosis. In the current study, we performed bone marrow punctures at two different locations, and also conducted multiple smear sample reading, and found that there were $0.5 \%$ suspicious juvenile cells. Therefore, we suggest to examine the entire smear sample, and if suspicious juvenile cells are found, further examinations and consultations should be done.

Acute lymphocytic leukemia, on the other hand, is a clonal malignant disease originate from lymphocytes, with extremely hyperplasia of the bone marrow and mainly comprised of primitive cells, making it very different from aplastic anemia in bone marrow smear. In the current study, bone marrow smear results of the patient in the first two tests reached the diagnostic criteria of lymphocytic leukemia. Therefore, in patients with bone marrow hypoplasia, bone marrow smear should be performed, and if suspicious juvenile cells are found, we should follow up in the following time period, and flow cytometry as well as bone marrow biopsy should be done, especially immunological types should be determined by detecting the antigens, which can provide supplemental evidence for the diagnosis [14-18].

By analyzing the first flow cytometry results, we found that juvenile cells accounted for $0.69 \%$, which could be easily ignored during clinical diagnosis. The other results of this flow cytometry were in accordance with the juvenile cells. In the second test, we found that CD45percp became negative, which 
showed that maturity of this kind of lymphocytes were very low, and was different from most lymphoblasts and prolymphocytes. The other results further proved that this cell type was early-stage primitive cells, especially because CD34 was the symbol of hematopoietic stem cell [19-20]. Meanwhile, the high expression of CD10 showed that these lymphocytes originated from the germinal center.

\section{Conclusions}

For patients whose hypoplastic bone marrow suddenly became extremely hyperplasia, morphology was not very useful, thus definite diagnosis must be dependent on immunological types and bone marrow biopsy results.

\section{Abbreviations}

WBC: peripheral white blood cells

RBC: red blood cells

HGB: hemoglobin

PLT: platelet

NRBC: nucleated red blood cells

\section{Declarations}

\section{Ethics approval and consent to participate}

The current study has been approved by the Ethics Committee of The First Affiliated Hospital of Hebei North University, and the patientd signed written informed consent.

\section{Consent for publication}

Not applicable.

\section{Availability of data and materials}

The datasets used and/or analysed during the current study are available from the corresponding author on reasonable request.

\section{Conflict of Interest Statement}

The authors have no conflicts of interest to declare.

\section{Funding Sources}


Zhangjiakou science and technology project. No.1811032D-6.

\section{Author Contributions}

Substantial contributions to the conception or design of the work》ZB,CY,WX

Acquisition, analysis, or interpretation of data for the work囚LX,FS,ZS

Drafting the work or revising it critically for important intellectual content $₫ Z F, G D, G L$

Final approval of the version to be published $\nabla$ All Authors

\section{Acknowledgements}

Not applicable.

\section{References}

1. Amarilla SP, Gómez-Laguna J, Carrasco L, Rodríguez-Gómez IM, Caridad Y Ocerín JM, Graham SP, et al. Porcine reproductive and respiratory syndrome type 1 viruses induce hypoplasia of erythroid cells and myeloid cell hyperplasia in the bone marrow of experimentally infected piglets independently of the viral load and virulence. Vet Microbiol. 2017;201凶126-135.

2. Wang L, Liu H. Pathogenesis of aplastic anemia. Hematology. 2019;24(1) §559-566.

3. Moore CA, Krishnan K. Aplastic Anemia. 2019 May 14. In $₫$ StatPearls [Internet]. Treasure Island (FL) $\bigotimes$ StatPearls

4. Scharff BFSS, Modvig S, Marquart HV, Christensen C. Integrin-Mediated Adhesion and Chemoresistance of Acute Lymphoblastic Leukemia Cells Residing in the Bone Marrow or the Central Nervous System. Front Oncol. 2020;10ख775.

5. Patel AA, Thomas J, Rojek AE, Stock W. Biology and Treatment Paradigms in T Cell Acute Lymphoblastic Leukemia in Older Adolescents and Adults. Curr Treat Options Oncol. 2020;21(7) $\llbracket 57$.

6. Gavralidis A, Brunner AM. Novel Therapies in the Treatment of Adult Acute Lymphoblastic Leukemia. Curr Hematol Malig Rep. 2020. doi区 10.1007/s11899-020-00591-4.

7. Caraccio C, Krishna S, Phillips DJ, Schürch CM. Bispecific Antibodies for Multiple Myeloma $\triangle A$ Review of Targets, Drugs, Clinical Trials, and Future Directions. Front Immunol. 2020;11ه501.

8. Brodsky RA, Jones RJ. Aplastic anaemia[J]. Lancet, 2005, 365 (9471)ه1647 -1656.

9. Ohad Etzion ,Varun Takyar, Victor \ovack, et al囚Spleen and Liver Volumetrics as Surrogate Markers of Hepatic Venous Pressure Gra-dient in Patients With Noncirrhotic Portal Hypertension [J]. Hepatol Commun,2018,2(8) $₫ 919-928 \rrbracket$

10. Fattizzo B, Levati G, Cassin R, Barcellini W. Eltrombopag in Immune Thrombocytopenia, Aplastic Anemia, and Myelodysplastic Syndrome囚 From Megakaryopoiesis to Immunomodulation. Drugs. 2019;79(12)ه1305-1319. 
11. Liu C, Sun Y, Shao Z. Current Concepts of the Pathogenesis of Aplastic Anemia. Curr Pharm Des. 2019;25(3) $₫ 236-241$.

12. de Latour RP, Risitano A, Dufour C. Severe Aplastic Anemia and PNH. In $\bowtie$ Carreras E, Dufour C, Mohty M, Kröger N, editors. The EBMT Handbook囚 Hematopoietic Stem Cell Transplantation and Cellular Therapies [Internet]. 7th ed. Cham (CH)凶 Springer; 2019. Chapter 77.

13. Babushok DV. A brief, but comprehensive, guide to clonal evolution in aplastic anemia. Hematology Am Soc Hematol Educ Program. 2018;2018(1)\457-466.

14. Boddu PC, Kadia TM. Molecular pathogenesis of acquired aplastic anemia. Eur J Haematol. 2019;102(2) $ه 103-110$.

15. Buechele C, Breese EH, Schneidawind D, Lin CH, Jeong J, Duque-Afonso J, et al. MLL leukemia induction by genome editing of human CD34+ hematopoietic cells[J]. Blood, 2015, 126(14): 16831694. DOIه10.1182/blood-2015-05- 646398.

16. El Chaer F, Keng M, Ballen KK. MLL-Rearranged Acute Lymphoblastic Leukemia. Curr Hematol Malig Rep. 2020;15(2)ष83-89.

17. Makishima H. [Clonal hematopoiesis in aplastic anemia]. Rinsho Ketsueki. 2018;59(10)ه1962-1968.

18. DeZern AE, Brodsky RA. Haploidentical Donor Bone Marrow Transplantation for Severe Aplastic Anemia. Hematol Oncol Clin North Am. 2018;32(4) 『629-642.

19. Hughes MR, Hernaez DC, Cait J, Refaeli I, Lo B, Roskelley C, et al. A sticky wicket:Defining molecular functions for CD34 in hematopoietic cells. Exp Hematol. 2020『S0301-472X(20)30181-8.

20. Smith TJ. Potential Roles of CD34+ Fibrocytes Masquerading as Orbital Fibroblasts in ThyroidAssociated Ophthalmopathy. J Clin Endocrinol Metab. 2019;104(2) $₫ 581-594$.

\section{Figures}



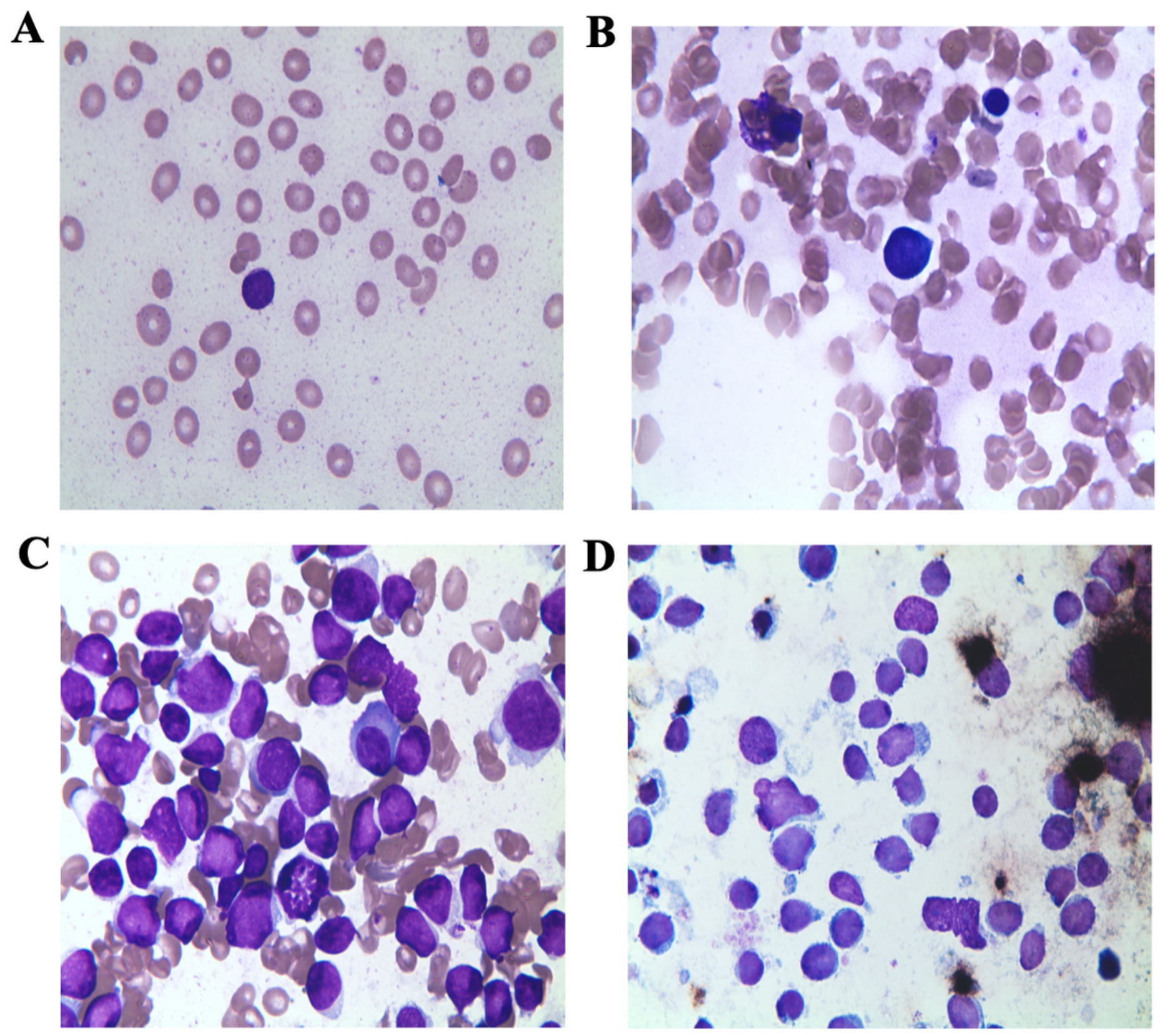

\section{Figure 1}

A. The first bone marrow smear result. It shows that mature lymphocytes (wright stain $\times 1000$ ) were regularly shaped, with little and bright blue cytoplasm, densificated chromatin and regular cell nucleus, no nucleolus was seen. B. The second bone marrow smear result. It shows the rarely seen suspicious juvenile cells (wright stain $\times 1000$ ), whose shape was regular and slightly large, with little and bright blue cytoplasm, loosen chromatin and regular cell nucleus, no nucleolus was seen. C. The third bone marrow smear result. It shows very active juvenile cells, whose shape was regular and slightly large, with little and bright blue cytoplasm, loosen chromatin and regular cell nucleus, and also had obvious nucleolus. C. POX staining in the third bone marrow smear, which was negative. 


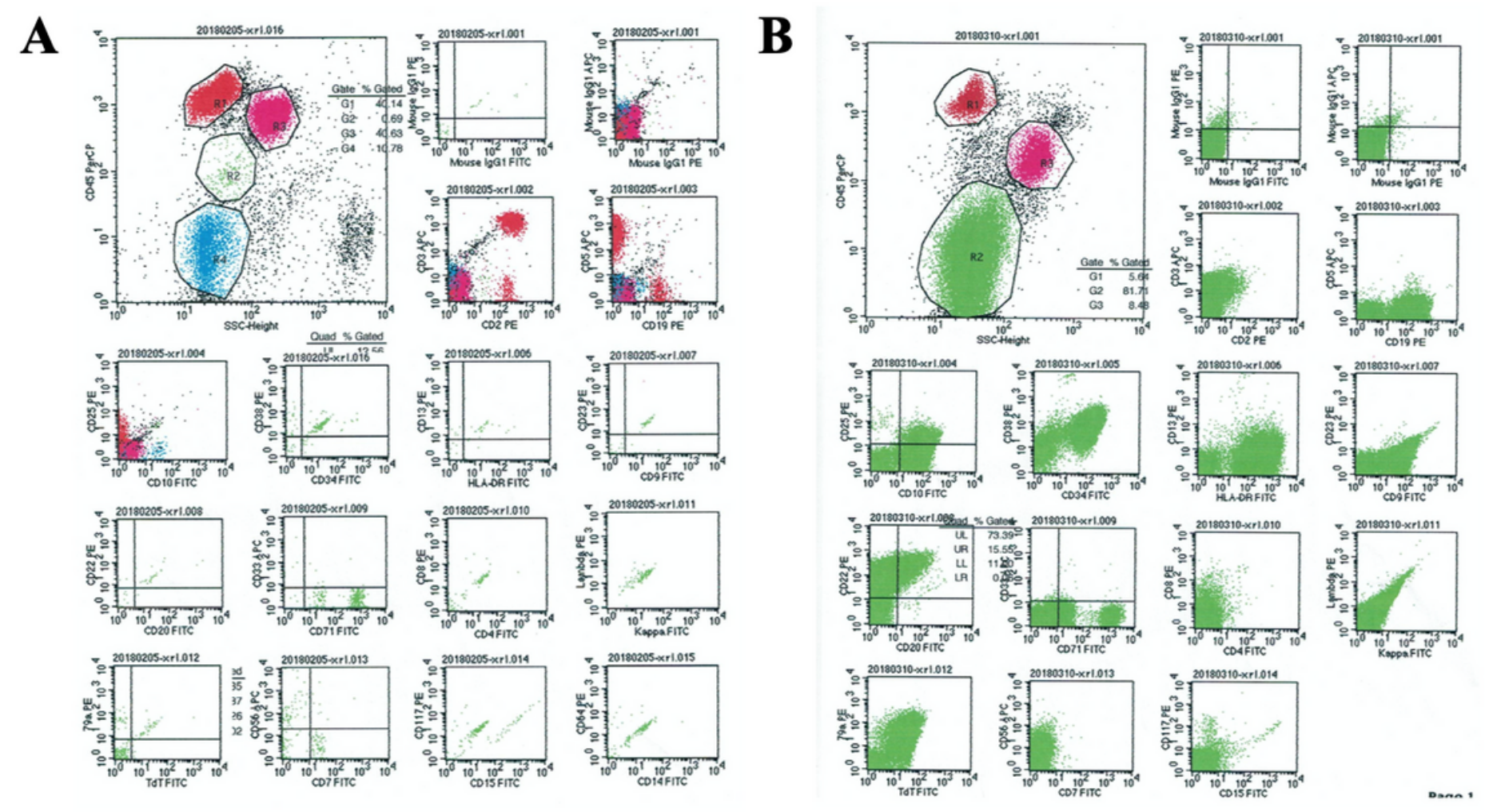

Figure 2

Immunological types of the bone marrow. A. The test done on 20180205. Abnormal cells accounted for $0.69 \%$, expression of CD45percp was weak, while SSC was slightly strong. Expression of CD34 (27.48\%区,

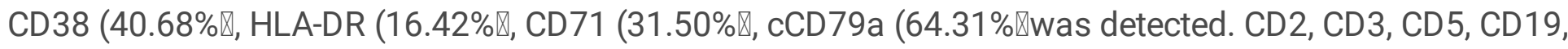
CD10, CD20, CD22, CD9, CD4, CD8, Kappa, Lambda, CD56, CD117, CD7, CD13, CD33, CD64, CD14, MP0, CD23 was not expressed. B. The test done on 201803105. Abnormal cells accounted for $81.71 \%$, CD45percp was not expressed, and SSC was weakly expressed. Expression of CD34, CD38, CD19, CD10, CD9, CD22, HLA-DR, CD71, cCD79a, CD5 was detected. CD2, CD3, CD5, CD20, CD4, CD8, Kappa, Lambda, CD56, CD117, CD7, CD13, CD33, CD64, CD14, MPO, CD23 was not expressed.

\section{Supplementary Files}

This is a list of supplementary files associated with this preprint. Click to download.

- CAREchecklistEnglish2013.pdf 